\title{
RECENT PROGRESS IN COMPUTATIONAL AND APPLIED PDES
}




\section{RECENT PROGRESS IN COMPUTATIONAL AND APPLIED PDES}

Conference Proceedings for the International Conference Held in Zhangjiajie in July 2001

Edited by

TONY F. CHAN

University of California at Los Angeles

Los Angeles, California

YUNQING HUANG

Xiangtan University

Xiangtan City of Hunan, China

TAO TANG

Hong Kong Baptist University

Hong Kong, China

JINCHAO XU

Pennsylvania State University University Park, Pennsylvania

and

LONG-AN YING

Peking University

Beijing, China

Springer Science+Business Media, LLC 
Proceedings of the International Symposium on Computational and Applied PDEs, held July 1-7, 2001, in Zhangjiajie National Park, China

ISBN 978-1-4613-4929-7 ISBN 978-1-4615-0113-8 (eBook)

DOI 10.1007/978-1-4615-0113-8

(C)2002 Springer Science+Business Media New York

Originally published by Kluwer Academic/Plenum Publishers, New York in 2002

Softcover reprint of the hardcover 1st edition 2002

http://www.wkap.com

$\begin{array}{llllllllll}10 & 9 & 8 & 7 & 6 & 5 & 4 & 3 & 2 & 1\end{array}$

A C.I.P. record for this book is available from the Library of Congress

All rights reserved

No part of this book may be reproduced, stored in a retrieval system, or transmitted in any form or by any means, electronic, mechanical, photocopying, microfilming, recording, or otherwise, without written permission from the Publisher, with the exception of any material supplied specifically for the purpose of being entered and executed on a computer system, for exclusive use by the purchaser of the work 


\section{Contents}

Preface ix

List of participants $\quad$ xi

Shift Theorems for the Biharmonic Dirichlet Problem 1

Constantin Bacuta, James H. Bramble, Joseph E. Pasciak

Weizhu Bao, Shi Jin, Peter A. Markowich

Inverse Doping Problems for Semiconductor Devices

Martin Burger, Heinz W. Engl, Peter A. Markowich

Superconductivity Analogies, Ferroelectricity and Flow Defects in Liquid Crystals 55 M. Carme Calderer

Bayesian Inpainting Based on Geometric Image Models

Tony F. Chan, Jianhong Shen

A Combination of Algebraic Multigrid Algorithms with the Conjugate Gradient Technique Qianshun Chang and Zhaohui Huang

Basic Structures of Superconvergence in Finite Element Analysis

Chuanmiao Chen

A Posteriori Error Estimates for Mixed Finite Elements of a Quadratic Control Problem Yanping Chen, Wenbin Liu

Superconvergence of Least-Squares Mixed Finite Element Approximations over Quadrilaterals Yanping Chen, Manping Zhang

A Posteriori Error Analysis and Adaptive Methods for Parabolic Problems 
Numerical Computation of Quantized Vortices in the Bose-Einstein Condensate 157 Qiang Du

An Optimization Algorithm for the Meteorological Data Assimilation Problem 171 Eva Eggeling, Shlomo Ta'asan

Analytic Aspects of Yang-Mills Fields

Gang Tian

The Shape Optimization of Axisymmetric Structures Based on Fictitious Loads Variable

Shuguang Gong, Yunqing Huang, Guilan Xie, Bo Hong

A New Kind of Preconditioner for Interface Equations of Mortar Multipliers on Subspaces Qiya $\mathrm{Hu}$

An Optimal Error Estimate for an h-p Clouds Galerkin Method

Jun Hu, Yunqing Huang, Weimin Xue

Some Problems in Large Scale Non-Hermitian Matrix Computations

Zhongxiao Jia

Global Propagation of Regular Nonlinear Hyperbolic Waves

Tatsien $\mathrm{Li}$

Numerical Simulation of 3D Shallow Water Waves on Sloping Beach

Tiejun Li, Pingwen Zhang

High Performance Finite Element Methods

Qun Lin

Algebraic Multigrid Method On Lattice Block Materials

Shi Shu, Jinchao Xu, Yingxiong Xiao, Ludmil Zikatanov

On the Existence of Symmetric Three Dimensional Finger Solutions

Jianzhong Su and Bao Loc Tran

A Combined Mixed Finite Element and Discontinuous Galerkin Method for Miscible Displacement Problem in Porous Media

Shuyu Sun, Béatrice Rivière and Mary F. Wheeler

Numerical Simulation and Coarse-Graining of Large Particle Systems

Shlomo Ta'asan

Modeling and Simulations for Electrochemical Power Systems

Jinbiao Wu, Jinchao Xu

On the Error Estimates of the Fully Discrete Nonlinear Galerkin Method with Variable Modes to Kuramoto-Sivashinsky Equation 
Wu Yu-jiang, Yang Zhong-hua

A Perturbed Density-Dependent Navier-Stokes Equation Yuelong Xiao

A Cascadic Multigrid Method for Solving Obstacle Problems

J. P. Zeng, S. Z. Zhou, J. T. Ma

The Fundamental Equation of Two-Dimensional Layer Flows of the Melt Feedstock in the Powder Injection Molding Process

Zhoushun Zheng, Xuanhui Qu

Index 


\section{Preface}

The International Symposium on Computational \& Applied PDEs was held at Zhangiiajie National Park of China from July 1-7, 2001. The main goal of this conference is to bring together computational, applied and pure mathematicians on different aspects of partial differential equations to exchange ideas and to promote collaboration. Indeed, it attracted a number of leading scientists in computational PDEs including Doug Arnold (Minnesota), Jim Bramble (Texas A \& M), Achi Brandt (Weizmann), Franco Brezzi (Pavia), Tony Chan (UCLA), Shiyi Chen (John Hopkins), Qun Lin (Chinese Academy of Sciences), Mitch Luskin (Minnesota), Tom Manteuffel (Colorado), Peter Markowich (Vienna), Mary Wheeler (Texas Austin) and Jinchao Xu (Penn State); in applied and theoretical PDEs including Weinan E (Princeton), Shi Jin (Wisconsin), Daqian Li (Fudan) and Gang Tian (MIT). It also drew an international audience of size 100 from Austria, China, Germany, Hong Kong, Iseael, Italy, Singapore and the United States.

The conference was organized by Yunqing Huang of Xiangtan University, Jinchao Xu of Penn State University, and Tony Chan of UCLA through ICAM (Institute for Computational and Applied Mathematics) of Xiangtan university which was founded in January 1997 and directed by Jinchao Xu. The scientific committee of this conference consisted of Randy Bank of UCSD, Tony Chan of UCLA, K. C. Chang and Long-an Ying of Peking University, Qun Lin, ZhongCi Shi and Yaxiang Yuan of Chinese Academy of Sciences, Gang Tian of MIT, Mary Wheeler of UT Austin, Jinchao Xu of Penn State, and Yulin Zhou of Institute for Applied Physics and Computational Mathematics, Beijing.

The one-week conference featured 20 invited speakers, each of whom gave 45-minutes lectures, and about 40 other speakers. All invited talks were of high quality, covering several aspects of modern computational and theoretical partial differential equations. The conference site Zhangiajie is the top one or two national park in China. It is located in Hunan Province. Participants visiting Zhangjiajie the first time were impressed by the beautiful view of the 
mountain, lake and canions. More details on the conference can be found in http://www.math.psu.edu/ccma/pde2001.

We would like to thank Professor Shucheng Li, Preseident of Xiangtan University, for his support and also for his participating the openning ceremony. Thanks also go to Professor Jiping Zhang, Dean of School of Mathematical Sciences at Peking University for his kind support to this conference. Moreover, the conference received considerable financial supports. The main grants were provided by the Institute for Computational and Applied Mathematics of Xiangtan University, School of Mathematical Sciences of Peking University, the Center for Computational Mathematics and Applications of Penn State University, the Science and Technology Department of Hunan Province, the National Science Foundation of China, and the State Key Basic Research Project "Large Scale Scientific Computing Research". We are grateful to all sponsors for their generous support.

These conference proceedings were refereed. We would like to thank all referees for their support. The performance of the meeting depended very much on many helpers, including Susan $\mathrm{He}$, Xu Chen, Zhongbo Chen, Jianmei Yuan and Qishen Xiao of Xiangtan University and Rosemary Manning of Penn State University. We appreciate their assistance in making the conference organization a success. Finally, we thank Tammy Lam and Amy Lee of Hong Kong Baptist University for the considerable work they put into producing the final layout of this proceedings.

Editors:

T.F. Chan, UCLA

Y.-Q. Huang, XTU

T. Tang, HKBU

J.-C. Xu, Penn State

L.-A. Ying, PKU.

June 2002 


\section{List of participants}

Shidfar Abdullah, shidfar@iust.ac.ir

Department of Mathematics, Iran University of Science and Technology

Douglas N. Arnold, dna@math.psu.edu; arnold@ima.umu.edu

Department of Mathematics, Penn State University

Steven F. Ashby, sfashby@llnl.gov

Center for Applied Scientific Computing, Lawrence Livermore National Laboratory

James H. Bramble, bramble@math.tamu.edu; barth@nas.nasa.gov

Mathematics Department, Texas A\&M University

Achi Brandt, achi@wisdom.weizmann.ac.il

Department of Computer Science \& Applied Mathematics, The Weizmann Institute of Science

Franco Brezzi, brezzi@ian.pv.cnr.it; brezzi@dragon.ian.pv.cnr.it

Dipartimento di Matematica, Universita' di Pavia

Qingdong Cai, caiqd@mech.pku.edu.cn

Department of Mechanical Engineering, Peking University

Xiaolin Cao, xiaolincao@ 163.com

School of Computer Science, National University of Defense Technology, China

Maria-Carme Calderer, mcc@math.psu.edu

Department of Mathematics, Penn State University

Tony Chan, chan@ipam.ucla.edu

Institute for Pure and Applied Mathematics (IPAM), UCLA

Chuanmiao Chen, cmchen@mail.hunnu.edu.cn

Department of Mathematics, Hunan Normal University, China

Jinru Chen, jrchen@pine.njnu.edu.cn

School of Mathematical Sciences, Nanjing Normal University, China

Shiyi Chen, syc@taylor.me.jhu.edu

Department of Mechanical Engineering, The Johns Hopkins University

\section{Yixin Chen}

Scientific Publishing Company of Hunan, China

Yanping Chen, ypchen@xtu.edu.cn

Department of Mathematics, Xiangtan University, China

Zhiming Chen, zmchen@Isec.cc.ac.cn

Institute of Computational Mathematics, Chinese Academy of Sciences 
Yana Di Jbn58@water.pku.edu.cn

School of Mathematical Sciences, Peking University

Qiang Du, qdu@lsec.cc.ac.cn

Institute of Computational Mathematics, Chinese Academy of Sciences

Weinan E, weinan@math.princeton.edu; weinan@princeton.edu

Department of Mathematics, Princeton University

Eva Eggeling, eggeling@gmd.de; eva@ fleuler.gmd.de

Institute of Algorithms and Scientific Computing (SCAI), GMD-German National Research Center for Information Technology

Shuguang Gong, gongsg@xtu.edu.cn

Department of Mathematics, Xiangtan University, China

\section{Zhenting Hou}

Department of Mathematics, Railway Area of Central South University, China

Yunqing Huang, huangyq@xtu.edu.cn

Department of Mathematics, Xiangtan University, China

Zhaohui Huang, zhhuang@amath6.amt.ac.cn

Jun Hu, hujunlya@263.net

Department of Mathematics, Xiangtan University, China

Qiya Hu, hqy@Isec.cc.ac.cn

Institute of Computational Mathematics, Chinese Academy of Sciences

Zhongxiao Jia, zxjia@ dlut.edu.cn

Department of Applied Mathematics, Dalian University of Technology, China

Shi Jin, jin@math.wisc.edu

Department of Mathematics, University of Wisconsin-Madison

Daqian Li, dqli@fudan.edu.cn

Department of Mathematics, Fudan University

Qun Lin, qlin@staff.iss.ac.cn

Institute of Computational Mathematics, Chinese Academy of Sciences

Wenbing Liu, W.B.Liu@ukc.ac.uk

CBS, University of Kent

Mitchell Barry Luskin, luskin@math.umn.edu

School of Mathematics, University of Minnesota

Thomas A. Manteuffel, tmanteuf@colorado.edu

Department of Applied Mathematics, University of Colorado at Boulder 
Meng Mao, maomeng@263.net

Department of Soil and Water Sciences, China Agricultural University

Luisa Donatella Marini, marini@ian.pv.cnr.it; marini@dragon.ian.pv.cnr.it Dipartimento di Matematica and I.A.N.-C.N.R., Italy

Zeyao Mo,zy_mo@sina.com

Mo Mu,mamu@ust.hk

Department of Mathematics, Hong Kong University of Science \& Technology

Joseph E.Pasciak, pasciak@math.tamu.edu

Department of Mathematics, Texas A\&M University

Peter Markowich, peter.markowich@univie.ac.at

Institute of Mathematics, University of Vienna

Li Ren, renl@mx.cei.gov.cn

Department of Soil and Water Sciences, China Agricultural University

Xiumin Shao,shao@math03.math.ac.cn

Institute of Mathematics, Chinese Academy of Sciences,

Jie Shen, shen@math.psu.edu

Department of Mathematics, Penn State University

Ta'asan Shlomo,shlomo@andrew.cmu.edu; shlomo@sattva.math.cmu.edu Department of Mathematics Sciences, Carnegie Mellon University

Shi Shu, shushi@xtu.edu.cn

Department of Mathematics, Xiangtan University, China

Jianzhong Su, su@uta.edu

Department of Mathematics, University of Texas at Arlington

Yi Sun, ysun@pku.edu.cn

School of Mathematical Sciences, Peking University

Tao Tang, ttang@math.hkbu.edu.hk

Department of Mathematics, Hong Kong Baptist University

Zhijun Tan, tanzhijun1221@sina.com

Department of Mathematics, Xiangtan University, China

Gang Tian, tian@math.mit.edu

Department of Mathematics, MIT

Lihe Wang, lwang@math.uiowa.edu

Department of Mathematics, University of Iowa 
Ping Wang, pxw'10@psu.edu

Department of Mathematics, Penn State University

MaryWheeler,mfw@ticam.utexas.edu

Texas Institute for Computational and Applied Mathematics, University of Texas, Austin

Gabriel Christoph Wittum, wittum@iwr.uni-heidelberg.de

IFI, University Heidelberg

Haijun Wu,whj@Isec.cc.ac.cn

Morning Sight Center, Chinese Academy of Sciences

YujiangWu,myjaw@lzu.edu.cn

Department of Mathematics, Lanzhou University, China

Yuelong Xiao, Xiaoyl01@163.com

Department of Mathematics, Xiangtan University, China

Jie Xiao, Xiaojuan-xu@263.net

Department of Mathematics, Xiangtan University, China

Da Xu,daxu@hunnu.edu.cn

Department of Mathematics, Hunan Normal University, China

Jinchao Xu, xu@math.psu.edu

Department of Mathematics, Penn State University

Lung-an Ying, yingla@pku.edu.cn

School of Mathematical Sciences, Peking University

Harry Yserentant, harry@na.uni-tuebingen.de; yserentant@na.uni-tuebingen.de Mathematisches Institut, Universität Tubingen

\section{Haiyuan Yu}

Department of Mathematics, Xiangtan University, China

Ju'e Yang, chrysan@ china.com

Department of Mathematics, Xiangtan University, China

Ying Yang, y-y1976@263.net

Department of Mathematics, Xiangtan University, China

Jinping Zeng, blma@mail.hnu.net.cn; dhli@mail.hunu.edu.cn

Department of Applied Mathematics, Hunan University, China

Dakai Zhang, xiangshw@263.net ; zouaim@263.net

Department of Mathematics, Guizhou University, China

Deyue Zhang, zhangdeyue@263.net 
Morning Sight Center, Chinese Academy of Sciences

Manping Zhang, zhangmanping@ sina.com

Department of Mathematics, Xiangtan University, China

Pingwen Zhang, pzhang @ math.pku.edu.cn; pzhang @pku.edu.cn

School of Mathematical Sciences, Peking University

Shan Zhao, asan@cz3.nus.edu.sg

Department of Computational Science, National University of Singapore

Weiying Zheng, zhengweiying@sina.com

Peking University

Zhoushun Zheng, zszheng @ mail.csu.edu.cn

Department of Mathematics and Software, Central South University

Bin Zheng, bzheng@water.pku.edu.cn

School of Mathematical Sciences, Peking University

Huansong Zhou, hszhou@wipm.whenc.ac.cn

Institute of Physics \& Mathematics, Wuhan, Chinese Academy of Sciences

Yi Zhou, yizhou@fudan.ac.cn

Institute of Mathematics, Fudan University 